\title{
Massive current algebra in the many-flavor chiral Gross-Neveu model
}

\author{
Tamas Hauer* \\ Institute for Theoretical Physics \\ Lorand Eötvös University \\ H-1088 Budapest, Puskin u. 5-7, Hungary
}

\begin{abstract}
We study the algebra of $S U(n)$-currents in the many-flavor chiral Gross-Neveu model. The general structure of the current-current OPE leading to non-local quantum conserved charges is reviewed. We calculate the OPE in the one-flavor and the many-flavor models perturbatively and use renormalization group invariance to prove that our results are not altered by higher-order corrections. We conclude that in these models the non-local quantum charge exists which is the first step towards the proof of the absence of particle production and factorization.
\end{abstract}

PACS codes: 11.10.Kk 11.20.Ex 11.30.Na

keywords: non-local charge, operator product expansion, Gross-Neveu model, renormalization group

Address of correspondence:

Tamas Hauer

Institute for Theoretical Physics, Lorand Eötvös University

H-1088 Budapest, Puskin u. 5-7, Hungary

Tel: 36-1-266-7921; Fax: 36-1-266-0612

email: hauer@ludens.elte.hu

\footnotetext{
*E-mail: hauer@ludens.elte.hu
} 


\section{Introduction}

Two dimensional integrable systems - being exactly solvable - are ideal toy models for understanding the behavior of higher dimensional quantum field theories. In many cases a rich symmetry structure is hidden behind integrability and the existence of a sufficient number of conserved charges provides a non-perturbative definition of the model. These conservation laws can yield the determination of the exact S-matrix and form factors. A large class of massive integrable QFT's are characterized by infinite dimensional non-abelian quantum group symmetries (for a review see 11). The elements of the underlying Yangian algebra are conserved charges which may be obtained as spectral coefficients of the quantum monodromy operator [2, 3], alternatively the generators can be defined as nonlocal functions of the local symmetry currents. The non-perturbative definition of such a non-local charge was first constructed by Lüscher in the non-linear $\sigma$-model [4]. Later other models were also investigated [5, 6, 7]. In all cases the short-distance expansion of the product of the local currents provides the key to the quantum conservation laws: different model-specific assumptions finally lead to the theorem proved in Lüscher's original paper. Possible generalizations to higher dimensions is discussed in [8, 9].

As it was pointed out by Bernard [5] the origin of the Yangian symmetry in the quantum theory is the quantum version of the curl-free equation of the local currents:

$$
\partial_{0} j_{1}^{a}-\partial_{1} j_{0}^{a}+f^{a b c} j_{0}^{b} j_{1}^{c}=0 .
$$

If this field equation holds then the following expression defines a conserved quantity in the classical theory:

$$
Q_{1}^{a}=\frac{1}{4} \int_{-\infty}^{\infty} d y_{1} d y_{2} \epsilon\left(y_{1}-y_{2}\right) f^{a b c} j_{0}^{b}\left(t, y_{1}\right) j_{0}^{c}\left(t, y_{2}\right)+\int_{-\infty}^{\infty} d y j_{1}^{a}(t, y)
$$

In the quantum theory both of these equations are modified. One has to introduce an appropriate normal ordering to define the composite operator appearing 
in the curl-free equation which may lead to quantum corrections of this relation. The quantum non-local charge has to be defined using some regularization. The natural scheme is point-splitting which calls our attention to the short-distance behavior of the product of local operators. Indeed it turns out that it is the operator product expansion (OPE) of the local currents which should be viewed as the quantum analog of (1); it provides a natural normal-ordering and also the proper definition of the singular non-local charge.

Once we have been able to define the conserved $Q_{1}^{a}$ 's, the whole Yangian algebra can be generated by commutators of $Q^{a}$ 's and $Q_{1}^{a}$ 's [1]. The highly restrictive nature of this rich symmetry has been studied both in general and in specific models (see e.g. [11, 10]). Since the non-local charges do not commute with the Lorentz-boost operator, the Poincaré-algebra is no longer a direct multiplier of the symmetry. This allows one to deduce relations among the masses of the spectrum of particles (from the nontrivial comultiplication applied to multiparticle scattering) [12]. Also one can prove the absence of particle production and factorization which leads to the exact S-matrix [ [].

In this paper we study the above OPE. Our aim is to formulate a general criterion for the existence and conservation of the Lüscher-type non-local charge. Starting from the general OPE within the framework of asymptotically free theories, we find that the quantum counterpart of the non-local expression (2) exists and it is conserved if and only if the OPE contains no operators besides the current and its derivative. This is essentially the summary of the results of previous investigations. We first study how general principles constrain - besides the coefficient of the current and its derivatives - the presence and coefficients of possible extra operators. The general result is the following: apart from the current and its derivative, antisymmetric Lorentz-tensor operators may appear at the right hand side of the OPE with constant coefficients. This statement is important, because - as we will see in the concrete example - this makes it possible to decide whether a given operator is present or not. 
As an application of the general results we present our calculation in a special case, the multiflavor chiral Gross-Neveu model. Here we are able to derive exact OPE coefficients thus prove the conservation of the quantum non-local charge which generates the Yangian algebra. This proves that the multiflavor CGN is a concrete realization of Bernard's massive current algebra [0].

The plan of the paper is as follows. In section 2 we study the general features of the operator product $f^{a b c} j_{\mu}^{b}(x) j_{\nu}^{c}(0)$. We determine the most general form of the expansion. In section 3 we first show that the chiral Gross-Neveu model satisfies the requirements we set at the beginning of the general analysis then apply these results to it. We calculate the OPE perturbatively up to the first nontrivial order and argue that it is not modified at any higher order in perturbation theory.

\section{The operator product expansion}

The first part of this paper is devoted to the study of the Wilson-expansion of the product of local symmetry currents. After fixing the family of the $2 \mathrm{~d}$ models we plan to work in (which is intended to be general enough to include the formerly investigated theories) we deal with three questions: the operator content of the algebra, the functional form of the coefficients and the relation of these to the existence of the non-local charge. Examination of these points in detail yields, as a conclusion, the most general form of the expansion in the given family. We will show that concrete field theories are characterized by one scalar function and some constant numbers, and that the vanishing of the latter ensures the conservation of the non-local charge.

\subsection{The class of models}

The basis of the hierarchy of the non-local charges is the algebra of local currents, therefore we require a model to contain a set of local conserved currents, $j^{a \mu}(x)$, 
with the corresponding charges forming the following algebra:

$$
\begin{aligned}
Q^{a} & \equiv \int d x j^{a 0}(x, t) \\
{\left[Q^{a}, Q^{b}\right] } & =f^{a b c} Q^{c} .
\end{aligned}
$$

Here $f^{a b c}$ are structure constants of a semisimple Lie-algebra, gף. We also require that the $\mathrm{QFT}$ is renormalizable (to give meaning to the $\mathrm{OPE}$ ) and asymptotically free (to carry out dimensional analysis). Our main additional assumption is the following: In the adjoint representation of $\mathrm{g}$ the current $j_{\mu}^{a}(x)$ is the only operator of dimension $<2$. When composing this condition we kept the well known specific models in view and we think that their similar behavior can be traced back to this property. Examples are the $O(n)$ non-linear $\sigma$ models - where because of the constraint on the length of the isovector field new composite operators can be constructed by application of derivatives only - and Lagrangian fermionic theories - where the basic field is dimensionful. Another approach is the framework of the massive current algebras [5] where the smooth UV limit directly constrains the operator content.

From now on we will assume that these properties are valid in which case we can write down the most general form of our Wilson-expansion:

$$
\begin{aligned}
f^{a b c} j_{\mu}^{b}(x) j_{\nu}^{c}(0) & =C_{\mu \nu}^{\rho} j_{\rho}^{a}(0)+D_{\mu \nu}^{\sigma \rho}(x) \partial_{\sigma} j_{\rho}^{a}(0)+\ldots \\
C_{\mu \nu}^{\rho}(x) & =\mathcal{O}\left(|x|^{-1-0}\right) \\
D_{\mu \nu}^{\sigma \rho}(x) & =\mathcal{O}\left(|x|^{-0}\right)
\end{aligned}
$$

where ... include other terms of $\mathcal{O}\left(|x|^{-0}\right)$ and we denote with -0 the possible logarithmic (i.e. weaker than any power) corrections to the power-like singular behavior. In the following we will study the set of operators on the rhs and the general form of their coefficients by (following the idea of [⿴囗十) making use of general principles: discrete symmetries, locality, Lorentz-covariance and current conservation.

\footnotetext{
${ }^{1}$ Conventions: $f^{a b c *}=-f^{a b c} f^{a b c} f^{b c d}=-2 q \delta^{a d}$.
} 


\subsection{Operator content: discrete quantum numbers}

Let's denote charge conjugation (on the Hilbert space) by $C$, parity by $P$, and CPT by $\Theta$. The transformation properties of the current can be summarized as:

$$
\begin{aligned}
\Theta j_{\mu}^{a}(x, t) \Theta^{-1} & =-j_{\mu}^{a}(-x,-t) \\
\Theta \partial_{\mu} j_{\nu}^{a}(x, t) \Theta^{-1} & =\partial_{\mu} j_{\nu}^{a}(-x,-t) \\
P j_{\mu}^{a}(x, t) P^{-1} & =j_{\tilde{\mu}}^{a}(-x, t) \\
P \partial_{\mu} j_{\nu}^{a}(x, t) P^{-1} & =\partial_{\tilde{\mu}} j_{\tilde{\nu}}^{a}(-x, t) \\
C j_{\mu}^{a}(x, t) C^{-1} & =\beta^{a b} j_{\mu}^{b}(x, t) .
\end{aligned}
$$

Here tilde above a Lorentz index means multiplication by 1 or -1 if it is 0 or 1 , respectively. The algebra of the charges requires the following identities to hold for the matrix representing the charge conjugation:

$$
\begin{aligned}
\beta^{a b} \beta^{b c} & =\delta^{a c} \\
\beta^{a i} \beta^{b j} f^{i j c} & =f^{a b k} \beta^{k c} .
\end{aligned}
$$

Now consider the general expression for the OPE:

$$
f^{a b c} j_{\mu}^{b}(x) j_{\nu}^{c}(0)=\sum C_{\mu \nu}^{(i)}(x) O_{(i)}^{a}(0) .
$$

Here $O_{(i)}$ are linearly independent, hermitian operators. Application of the discrete symmetry transformations yields:

$$
\begin{aligned}
C O_{(i)}^{a}(x) C^{-1} & =\beta^{a b} O_{(i)}^{b}(x) \\
C_{\mu \nu}^{(i)}(x, t) \Theta O_{(i)}^{a}(0) \Theta^{-1} & =C_{\mu \nu}^{(i)}(-x,-t) O_{(i)}^{a}(0) \\
C_{\mu \nu}^{i}(x, t) P O_{i}^{a}(0) P^{-1} & =C_{\tilde{\mu} \tilde{\nu}}^{i}(-x, t) O_{i}^{a}(0) .
\end{aligned}
$$

The first equation shows that only operators with the same charge conjugation transformation character (identical to that of the current) may be present. The CPT and P symmetry involve the transformation of $x$, too, thus we get constraints on the coefficients in which the CPT-, and the P-charge of the corresponding operator appears. 


\subsection{The $\mathrm{x}$-dependence of the OPE coefficients}

In this section we will analyze the possible $\mathrm{x}$-dependent behavior of the OPEcoefficients. First we note that antihermicity of the lhs requires that the OPE coefficients of self-adjoint operators be pure imaginary. The analytic properties of these functions are determined by the spectrum condition which states that they are boundary values of complex functions analytic in $z=x-i y$ in the region $y^{2}>0, y^{0}>0$ [14].

As we will see in the next paragraphs the different operators do not influence each other's behavior under the general principles. The only exception is locality which mixes an operator with its derivative. However there is only one operator the derivative of which may appear due to our basic assumption. Therefore we start with examining the coefficients of the current and its derivative first and then we turn to the other operators.

CPT-symmetry From the equations (5) and (9) we get:

$$
\begin{aligned}
& C_{\mu \nu}^{\rho}(-x)=-C_{\mu \nu}^{\rho}(x) \\
& D_{\mu \nu}^{\sigma \rho}(-x)=D_{\mu \nu}^{\sigma \rho}(x) .
\end{aligned}
$$

Locality For spacelike $x$ the two operators commute: $f^{a b c}\left[j_{\mu}^{b}(x), j_{\nu}^{c}(0)\right]=0$ The operator product expansion of this equality reads:

$$
\begin{aligned}
0= & f^{a b c} j_{\mu}^{b}(x) j_{\nu}^{c}(0)+f^{a b c} j_{\nu}^{b}(0) j_{\mu}^{c}(x)= \\
= & \left(C_{\mu \nu}^{\rho}(x)+C_{\nu \mu}^{\rho}(-x)\right) j_{\rho}^{a}(0)+ \\
& \left(D_{\mu \nu}^{\sigma \rho}(x)+D_{\nu \mu}^{\sigma \rho}(-x)+x^{\sigma} C_{\nu \mu}^{\rho}(-x)\right) \partial_{\sigma} j_{\rho}^{a}(0)+O\left(|x|^{1-0}\right) .
\end{aligned}
$$

This immediately yields:

$$
C_{\nu \mu}^{\rho}(x)=C_{\mu \nu}^{\rho}(x) .
$$


To find the similar equation for $D_{\mu \nu}^{\sigma \rho}$ we divide the $\sigma \rho$-tensors into antisymmetric, symmetric traceless and trace parts:

$$
\begin{aligned}
D_{\mu \nu}^{\sigma \rho}(x)-\frac{1}{2} x^{\sigma} C_{\nu \mu}^{\rho}(-x) & =\bar{D}_{\mu \nu}^{\sigma \rho}(x)+\tilde{D}_{\mu \nu}^{\sigma \rho}(x)+\frac{1}{2} g^{\sigma \rho} D_{\mu \nu}(x) \\
\bar{D}_{\mu \nu}^{\sigma \rho}(x) & \equiv D_{\mu \nu}^{[\sigma \rho]}(x)-\frac{1}{2} x^{[\sigma} C_{\mu \nu}^{\rho]}(x) \\
\tilde{D}_{\mu \nu}^{\sigma \rho}(x) & \equiv D_{\mu \nu}^{\{\sigma \rho\}}(x)-\frac{1}{2} x^{\{\sigma} C_{\mu \nu}^{\rho\}}(x)-\frac{1}{2} g^{\sigma \rho} D_{\mu \nu}(x) \\
D_{\mu \nu}(x) & \equiv g_{\sigma \rho}\left(D_{\mu \nu}^{\sigma \rho}(x)-\frac{1}{2} x^{\sigma} C_{\mu \nu}^{\rho}(x)\right) .
\end{aligned}
$$

Current conservation implies that the trace part drops out from (13) and we obtain:

$$
\bar{D}_{\nu \mu}^{\sigma \rho}(x)+\tilde{D}_{\nu \mu}^{\sigma \rho}(x)=-\left(\bar{D}_{\mu \nu}^{\sigma \rho}(x)+\tilde{D}_{\mu \nu}^{\sigma \rho}(x)\right) .
$$

If there are other constraints on the tensor structure of the derivative of the current then this equation can be modified. For example in case of a free massless fermionic theory the conservation of the axial current implies that $\partial_{\sigma} j_{\rho}^{a}(x)$ is symmetric, in which case $\tilde{D}_{\nu \mu}^{\sigma \rho}(x)=-\tilde{D}_{\mu \nu}^{\sigma \rho}(x)$.

Lorentz-covariance Now we apply the previously found conditions to determine the most general Lorentz-tensor structure. One can construct tensors from $x^{\mu}$ and $g_{\mu \nu}$ :

$$
\begin{aligned}
& C_{\mu \nu}^{\rho}(x)=C_{1} g_{\mu \nu} x^{2} x^{\rho}+C_{2} x^{2}\left(x_{\mu} \delta_{\nu}^{\rho}+x_{\nu} \delta_{\mu}^{\rho}\right)+C_{3} x_{\mu} x_{\nu} x^{\rho} \\
& \tilde{D}_{\mu \nu}^{\sigma \rho}(x)=D_{S}\left(x^{\sigma}\left(x_{\mu} \delta_{\nu}^{\rho}-x_{\nu} \delta_{\mu}^{\rho}\right)+x^{\rho}\left(x_{\mu} \delta_{\nu}^{\sigma}-x_{\nu} \delta_{\mu}^{\sigma}\right)\right) \\
& \bar{D}_{\mu \nu}^{\sigma \rho}(x)=D_{A}\left(\delta_{\mu}^{\sigma} \delta_{\nu}^{\rho}-\delta_{\nu}^{\sigma} \delta_{\mu}^{\rho}\right) x^{2}
\end{aligned}
$$

where $C_{1}, C_{2}, C_{3}, D_{S}, D_{A}$ are scalar functions depending on $-x^{2}$. The $C$ 's are of order $|x|^{-4-0}$ while $D_{S}$ and $D_{A}$ both are of $|x|^{-2-0}$.

Current conservation We write down differential equations for the scalar functions defined above which are implied by current conservation. It is clear 
that in order the current be conserved the following equations have to hold:

$$
\partial^{\mu} C_{\mu \nu}^{\rho}=\partial^{\mu} D_{\mu \nu}^{\sigma \rho}=0 .
$$

It is straightforward to rewrite this equation in terms of the scalar coefficients. We get more elegant forms if we introduce new scalar functions which possess only logarithmic singularities:

$$
\begin{aligned}
C_{i}\left(-x^{2}\right) & \equiv \frac{\gamma_{i}\left(\log \left(-\mu^{2} x^{2}\right)\right)}{\left(-x^{2}\right)^{2}} \\
D_{S}\left(-x^{2}\right) & \equiv-\frac{1}{4} \frac{\delta_{S}\left(\log \left(-\mu^{2} x^{2}\right)\right)}{\left(-x^{2}\right)} \\
D_{A}\left(-x^{2}\right) & \equiv-\frac{1}{4} \frac{\delta_{A}\left(\log \left(-\mu^{2} x^{2}\right)\right)}{\left(-x^{2}\right)}
\end{aligned}
$$

where we introduced an arbitrary mass scale, $\mu$. The differential equations for these functions are:

$$
\begin{aligned}
\dot{\gamma}_{1}+\dot{\gamma}_{2}+\dot{\gamma}_{3}-\left(\gamma_{1}+\gamma_{2}\right) & =0 \\
2 \dot{\gamma}_{2}+\left(\gamma_{1}+\gamma_{2}\right) & =0 \\
2 \dot{\delta}_{S}+\left(\gamma_{1}+\gamma_{2}\right) & =0 \\
2 \delta_{S}+\left(\gamma_{1}+\gamma_{2}+\gamma_{3}\right) & =0 \\
2 \dot{\delta}_{A}-\gamma_{1}+\gamma_{2} & =0
\end{aligned}
$$

where dot above a function means differentiation with respect to the variable $\log \left(-\mu^{2} x^{2}\right)$.

The set of equations can be solved up to an undetermined function. First note that the combination

$$
2 \lambda \equiv \gamma_{1}+3 \gamma_{2}+\gamma_{3} .
$$

is constant. We can express $\gamma$ 's in terms of $\delta_{S}$ :

$$
\begin{aligned}
\gamma_{1} & =-2 \dot{\delta}_{S}-\delta_{S}-\lambda \\
\gamma_{2} & =\delta_{S}+\lambda \\
\gamma_{3} & =2 \dot{\delta}_{S}-2 \delta_{S},
\end{aligned}
$$


and we can write all the five coefficients in terms of one undetermined function, $\xi\left(\log \left(-\mu^{2} x^{2}\right)\right)$

$$
\begin{aligned}
\delta_{S} & =-\dot{\xi}-\lambda \\
\delta_{A} & =\dot{\xi}+\xi+\lambda .
\end{aligned}
$$

Note that all the model dependent information is encoded in $\xi$.

\subsection{Commutators of the conserved charges}

Before studying the OPE any further (i.e. the presence of operators not considered so far) we now verify that the operator product we have obtained this way is consistent with the algebra of the charges, defined in eq (3). We also want to relate the constant $\lambda$ to the normalization of the algebra. One can do this by calculating the (antisymmetrized) equal-time commutators of the currents, $j_{\mu}^{a}$ in terms of the invariant functions for small $\mathrm{x}$.

$$
f^{a b c}\left[j_{\mu}^{b}(x), j_{\nu}^{c}(0)\right]_{E T}=\lim _{\varepsilon \rightarrow 0} f^{a b c}\left(j_{\mu}^{b}(x,-i \varepsilon) j_{\nu}^{c}(0)-j_{\mu}^{b}(x, i \varepsilon) j_{\nu}^{c}(0)\right) .
$$

We substitute the scalar OPE-coefficients at argument $\left(-\varepsilon^{2}-x^{2}\right)$. The terms proportional to $\partial_{\mu} j_{\nu}^{a}$ are zero as $\varepsilon \rightarrow 0$ and the commutators become:

$$
\begin{aligned}
f^{a b c}\left[j_{0}^{b}(x), j_{0}^{c}(0)\right]_{E T} & =\lim _{\varepsilon \rightarrow 0}\left\{2 i \varepsilon\left(\left(C_{1}+2 C_{2}+C_{3}\right) \varepsilon^{2}+\left(C_{1}+2 C_{2}\right) x^{2}\right) j_{0}^{a}(0)\right\} \\
f^{a b c}\left[j_{0}^{b}(x), j_{1}^{c}(0)\right]_{E T} & =\lim _{\varepsilon \rightarrow 0}\left\{2 i \varepsilon\left(C_{2} \varepsilon^{2}+\left(C_{2}+C_{3}\right) x^{2}\right) j_{1}^{a}(0)\right\} \\
f^{a b c}\left[j_{1}^{b}(x), j_{1}^{c}(0)\right]_{E T} & =\lim _{\varepsilon \rightarrow 0}\left\{(-2 i \varepsilon)\left(C_{1} \varepsilon^{2}+\left(C_{1}+C_{3}\right) x^{2}\right) j_{0}^{a}(0)\right\} .
\end{aligned}
$$

We require the currents to be normalized according to (3):

$$
f^{a b c}\left[j_{0}^{b}(x), j_{\mu}^{c}(0)\right]_{E T}=-2 q \delta(x) j_{\mu}^{a}(0) .
$$

The normalization of the $C_{i}$ 's can be easily extracted by noting that the first and second commutator together yields:

$$
-4 q \delta(x)=\lim _{\varepsilon \rightarrow 0}\left\{\left(\gamma_{1}+3 \gamma_{2}+\gamma_{3}\right) \frac{2 i \varepsilon}{\varepsilon^{2}+x^{2}}\right\},
$$


which gives:

$$
\lambda=-\frac{q}{i \pi}
$$

Let's examine the commutator of the spacelike components of the current, too. Eq. (26) shows, that it is proportional to the timelike component. We introduce the (model dependent) constant $\omega$ with:

$$
f^{a b c}\left[j_{1}^{b}(x), j_{1}^{c}(0)\right]_{E T}=-2 q \omega \delta(x) j_{0}^{a}(0) .
$$

The two commutators involving $j_{1}^{a}$ yield:

$$
\begin{aligned}
-2 q(1+\omega) \delta(x) & =\frac{2 i \varepsilon}{\varepsilon^{2}+x^{2}}\left(\gamma_{2}-\gamma_{1}\right) \\
\omega & =\frac{-2}{\lambda} \dot{\delta}_{A}-1 .
\end{aligned}
$$

The scalar function $\delta_{A}$ is a possibly singular function of $\log \left(-\mu^{2} x^{2}\right)$. We expand it around $x=0$ as:

$$
\delta_{A}=d\left(\log \left(-\mu^{2} x^{2}\right)\right)^{p}+\mathcal{O}\left(\left(\log \left(-\mu^{2} x^{2}\right)\right)^{p-1+0}\right) .
$$

From (32) we see that different models fall into three categories. If $p>1$ then $\omega$ is infinite, the commutator of the spacelike components is not consistent with (30). If $p<1$ then $\dot{\delta_{A}}$ vanishes as $x \rightarrow 0$ and $\omega=-1$ while if $p=1$ then

$$
\omega=-2 \frac{d}{\lambda}-1
$$

\subsection{Lüscher's non-local charge}

We are now in the position to define the quantum counterpart of (2). The singularity is regularized by introducing a "point-splitting charge" 四:

$$
\begin{aligned}
Q_{\zeta}^{a}= & \int_{\left|y_{1}-y_{2}\right| \geq \zeta} d y_{1} d y_{2} \epsilon\left(y_{1}-y_{2}\right) f^{a b c} j_{0}^{b}\left(t, y_{1}\right) j_{0}^{c}\left(t, y_{2}\right)+ \\
& +Z(\zeta) \int_{-\infty}^{\infty} d y j_{1}^{a}(t, y) .
\end{aligned}
$$


The (divergent) renormalization $Z(\zeta)$ should be chosen so that the above expression gives a well-defined operator in the limit $\zeta \rightarrow 0$. It is clear that the divergence to be compensated is closely related to the singularity of the currentcurrent OPE. Moreover it turns out that the existence of the limit is not influenced by the presence of extra operators in the expansion and the proper choice for $Z(\zeta)$ involves the coefficients of the derivative of the current only:

$$
Z(\zeta)=\delta_{S}\left(\log \left(\mu^{2} \zeta^{2}\right)\right)-\delta_{A}\left(\log \left(\mu^{2} \zeta^{2}\right)\right)
$$

There remains, of course the question whether the operator defined this way is conserved. This can be answered by taking the time-derivative of the pointsplitting charge and examine if it vanishes or not in the $\zeta \rightarrow 0$ limit. Again we have to insert the OPE in the integral after which we find that if the renormalization constant $Z(\zeta)$ is defined as in $(36)$ then the contribution coming from those part of the OPE which involves the current and its derivative, vanishes as $\zeta \rightarrow 0$. On the other hand any operator which is independent of the current gives a nonvanishing result in this limit. This leads to the conclusion that the conservation of the non-local charge in the quantum theory depends on the existence of extra operators in the current-current OPE only.

\subsection{Coefficients of other operators}

After investigating the OPE coefficient of the current and its derivative the previous section provides enough motivation to turn to the question of what other operators can appear on the rhs of the expansion.

We showed that only those operators may be present which have the same $C$ parity as the current and due to our assumption that there is no operator with lower dimension than that of the derivative of the current, their coefficients are of order $O\left(|x|^{-0}\right)$.

Lorentz-scalar operators are ruled out since their coefficient should be proportional to $\varepsilon^{\mu \nu}$ - the only tensor which possesses antisymmetry, but parity is 
violated then. Pseudoscalar operators can occur, their coefficient must be proportional to $\varepsilon^{\mu \nu}$. For the coefficient, $G_{\mu \nu}^{\rho}(x)$ of a vector operator CPT and locality yield the same constraint as for that of the current but now it should be of dimension $O\left(|x|^{-0}\right)$. It is easy to see that there is no Lorentz-tensor of this kind composed from $g_{\mu \nu}$ and $x^{\mu}$ which satisfies the spectrum condition. The next simplest operators are the symmetric and antisymmetric tensors. The coefficient of a symmetric tensor can be described by one scalar function:

$$
B_{\mu \nu}^{\sigma \rho}(x)=B\left(x^{2}\right) x^{\{\sigma} x_{[\mu} \delta_{\nu]}^{\rho\}} .
$$

One can easily see that this satisfies the CPT condition and locality. However current conservation yields:

$$
\partial^{\mu} B_{\mu \nu}^{\sigma \rho}=\left(x^{\sigma} \delta_{\nu}^{\rho}+x^{\rho} \delta_{\nu}^{\sigma}\right)\left(2 x^{2} B^{\prime}+2 B\right)-4 x_{\nu} x^{\sigma} x^{\rho} B^{\prime}-2 x_{\nu} g^{\sigma \rho} .
$$

The only solution to this equation is the constant zero, the symmetric tensors are also ruled out. Let us now consider an antisymmetric tensor, the coefficient of which can again be described by a scalar function:

$$
E_{\mu \nu}^{\sigma \rho}(x)=E\left(x^{2}\right)\left(\delta_{\mu}^{\sigma} \delta_{\nu}^{\rho}-\delta_{\nu}^{\sigma} \delta_{\mu}^{\rho}\right)
$$

This satisfies the locality condition and CPT. The current conservation yields the following constraint on the functional form of $E$ :

$$
\partial_{\mu} E=0
$$

That is, any antisymmetric operator in our OPE must have constant coefficient. Note that the same holds for a pseudoscalar operator, since its coefficient must be proportional to $\varepsilon^{\mu \nu}$ and after redefining the operator so that it includes $\varepsilon^{\mu \nu}$ it becomes an antisymmetric tensor. Therefore the most general form of ... in (1) is a series of antisymmetric tensoroperators with constant coefficients.

\subsection{Summary}

In this section we have gathered all the information about the current-current OPE which are valid model-independently. The individual theories are char- 
acterized by the function $\xi$ and the constants $E_{i}$ belonging to operators $O_{i}$ as follows:

$$
\begin{gathered}
f^{a b c} j_{\mu}^{b}(x) j_{\nu}^{c}(0)= \\
\left(\gamma_{1} \frac{g_{\mu \nu} x^{\rho}}{x^{2}}+\gamma_{2} \frac{x_{\mu} \delta_{\nu}^{\rho}+x_{\nu} \delta_{\mu}^{\rho}}{x^{2}}+\gamma_{3} \frac{x_{\mu} x_{\nu} x^{\rho}}{x^{4}}\right)\left(j_{\rho}^{a}(0)+\frac{x^{\sigma}}{2} \partial_{\sigma} j_{\rho}^{a}(0)\right)+ \\
\left(\frac{\delta_{S}}{4} \frac{x_{\mu} x^{\rho} \delta_{\nu}^{\sigma}+x_{\mu} x^{\sigma} \delta_{\nu}^{\rho}-x_{\nu} x^{\rho} \delta_{\mu}^{\sigma}-x_{\nu} x^{\sigma} \delta_{\mu}^{\rho}}{x^{2}}+\frac{\delta_{A}}{4}\left(\delta_{\mu}^{\sigma} \delta_{\nu}^{\rho}-\delta_{\nu}^{\sigma} \delta_{\mu}^{\rho}\right)\right) \partial_{\sigma} j_{\rho}^{a}(0) \\
+\sum_{i} E_{i} O_{[\mu \nu]}^{i}(0)
\end{gathered}
$$

with

$$
\begin{aligned}
\gamma_{1} & =-2 \dot{\delta}_{S}-\delta_{S}+\frac{q}{i \pi} \\
\gamma_{2} & =\delta_{S}-\frac{q}{i \pi} \\
\gamma_{3} & =2 \dot{\delta}_{S}-2 \delta_{S} \\
\delta_{S} & =-\dot{\xi}+\frac{q}{i \pi} \\
\delta_{A} & =\dot{\xi}+\xi-\frac{q}{i \pi} \\
E_{i} & =\text { constant. }
\end{aligned}
$$

The interesting models are in which no operators other than the current and its derivative appear in the OPE. In these cases a non-local conserved quantum charge can be defined as in (35), (36).

As the first example for the above we recall the $O(n)$ non-linear $\sigma$-model. The leading behavior of the OPE coefficients is the following 顿:

$$
\begin{aligned}
\gamma_{1}= & -\frac{\lambda}{2} \quad \gamma_{2}=\frac{\lambda}{2} \quad \gamma_{3}=\lambda \\
\delta_{S} & =-\frac{\lambda}{2} \\
\delta_{A} & =-\frac{\lambda}{2}\left(1+\log \left(-\mu^{2} x^{2}\right)\right) \\
\omega & =0 .
\end{aligned}
$$


Thus in the $O(n)$-models $\xi=-\lambda\left(1+\frac{1}{2} \log \left(-\mu^{2} x^{2}\right)\right)$. (As it was pointed out by Lüscher 13 there must be subleading $(\log \log ())$ singularities too.) The parameter $\mu$ is proportional to to the mass of the particles forming the $n$-plet. From the singularity of $\delta_{A}, p=1$ (see (33)) and substituting it into (34) we get $\omega=0$. This is exactly the expected behavior: the spacelike components of the current are constructed out of the spacelike derivatives of the basic scalar field and these necessarily commute.

Now let us see how the construction based on the massive current algebra [0] fits in this picture. Here the assumption is that the smooth UV-limit of the currents leads to the corresponding Kac-Moody algebra i.e. clearly no operator can appear in the OPE. Following [5] the OPE-coefficients of the models belonging to this category:

$$
\begin{aligned}
\gamma_{2} & =-\gamma_{1}=\lambda \quad \gamma_{3}=0 \\
\delta_{S} & =0 \\
\delta_{A} & =-\lambda \log \left(-\mu^{2} x^{2}\right) \\
\omega & =1 .
\end{aligned}
$$

Here again only the leading singularity is shown. One can see that $\xi=-\lambda \log \left(-\mu^{2} x^{2}\right)$, ( $\mu$ is a model-dependent mass parameter), again $p=1$, which now leads to $\omega=1$, that is

$$
f^{a b c}\left[j_{1}^{b}(x), j_{1}^{c}(0)\right]_{E T}=-2 q \delta(x) j_{0}^{a}(0) .
$$

As a concrete example for these algebras Bernard studies the $S O(n)$ GN-models - assuming that these really belong to this family. In the remaining part we deal with the $S U(n)$ multiflavor chiral GN-models and prove that they are concrete realizations of the massive current algebra framework. 


\section{The many-flavor chiral Gross-Neveu model}

In the second part of this paper we will give an example for the above results in a concrete Lagrangian quantum field theory. Our choice is the (one- and) many-flavor chiral Gross-Neveu model (CGN) [15], these theories possess the set of conserved local currents with the algebra (3) so one may expect to be able to define a non-local conserved charge. This is certainly the case for the one-flavor model where both the Ward identities corresponding to the non-local symmetry [16] and the explicit construction of the quantum charge [7] have been studied. As we saw in the previous section the sufficient condition for the existence of the quantum charge is that only the currents and their derivatives appear in our OPE. This is what we will prove in the following. Our strategy is: we allow all the possible operators on the rhs of the OPE which can be constructed from the basic fermionic field with the right quantum numbers and dimension. Then we show that either the corresponding coefficients are zero or the operator is not linearly independent of the current.

\subsection{The Lagrangian theory of the CGN model}

The chiral Gross-Neveu (CGN) model [15] is one of the most investigated toymodels. It is asymptotically free 17, the masses of the particle spectrum are generated dynamically 15. The continuous chiral symmetry is unbroken: the massive particles do not carry chiral charge 18$]$. The extension of the model to the multiflavor case is motivated by the equivalence of the $N_{\text {flavors }} \rightarrow \infty$ limit and the principal $S U(n)$ chiral model $[19,20]$. Two-loop renormalizability of the multiflavor model is proven in [21.

Though there are indications that there is much difference between the one- and the multiflavor models they can be treated similarly from the perturbative point of view. Therefore in the following section we will speak about the many-flavor model without restricting ourselves to the number of flavors greater than one 
case. The basic field is a massless fermion with a color and a flavor degree of freedom:

$$
\psi(x) \equiv \psi^{I i}(x),
$$

where $I$ runs from 1 to $N$ and transforms under the fundamental representation of $S U(N)_{f}$ and $i$ runs from 1 to $n$ and transforms under the fundamental representation of $S U(n)_{c}$. There are four types of vector currents which can be built as bilinears of the basic field. These are:

$$
\begin{aligned}
j^{\mu}(x) & \equiv \bar{\psi}(x) \gamma^{\mu} \psi(x) \\
j_{\mu}^{a}(x) & \equiv \bar{\psi}(x) \lambda^{a} \gamma_{\mu} \psi(x) \\
j_{\mu}^{A}(x) & \equiv \bar{\psi}(x) \Lambda^{A} \gamma_{\mu} \psi(x) \\
j_{\mu}^{A a}(x) & \equiv \bar{\psi}(x) \lambda^{a} \Lambda^{A} \gamma^{\mu} \psi(x) .
\end{aligned}
$$

Here $\lambda^{a}$ and $\Lambda^{A}$ are the generators of the color and flavor group in the fundamental representation 7 . The Minkowskian action is:

$$
S=\int d^{2} x\left(\bar{\psi} i \not \partial \psi-\frac{g^{2}}{2} j_{\mu}^{a} j^{a \mu}\right) .
$$

As far as the operator content is concerned the building block is the fundamental Fermi field. The main condition holds: in the adjoint representation of $S U(n)_{c}$ the color current is the only flavor-singlet, dimension=1 operator. In the general OPE operators with canonical dimension $=2$ can have nonvanishing coefficients, among these there are bilinear and quadrilinear expressions of the $\psi$-field. Now we list these.

There are two bilinear, dimension $=2$ operators, they are the derivative of the color current - which we expect to have - and an antisymmetric derivative of the basic fields (which we want to get rid of):

$$
\begin{gathered}
\partial_{\nu} j_{\mu}^{a}(x) \equiv \partial_{\nu} \bar{\psi} \lambda^{a} \gamma_{\nu} \psi+\bar{\psi} \lambda^{a} \gamma_{\mu} \partial_{\nu} \psi \\
k_{\mu \nu}^{a}(x) \equiv i\left(\partial_{\mu} \bar{\psi} \lambda^{a} \gamma_{\nu} \psi-\bar{\psi} \lambda^{a} \gamma_{\nu} \partial_{\mu} \psi\right) . \\
{ }^{2} \text { conventions: }\left[\lambda^{a}, \lambda^{b}\right]=f^{a b c} \lambda^{c} f^{a b c *}=-f^{a b c} \quad f^{a b c} f^{b c d}=-2 q \delta^{a d}
\end{gathered}
$$


There are several quadrilinear operators. The chiral symmetry allows the fol-

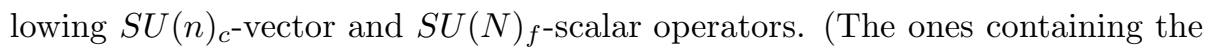
unit in Dirac-space or $\gamma_{5}$ are either ruled out by chiral symmetry or can be Fierz-transformed into the following forms)

$$
\begin{aligned}
l_{\mu \nu}^{a}(x) & \equiv\left(\bar{\psi} \gamma_{\mu} \psi\right)\left(\bar{\psi} \lambda^{a} \gamma_{\nu} \psi\right)(x) \\
N_{\mu \nu}^{a}(x) & \equiv i f^{a b c}\left(\bar{\psi} \gamma_{\mu} \lambda^{b} \psi\right)\left(\bar{\psi} \gamma_{\nu} \lambda^{c} \psi\right)(x) \\
n_{\mu \nu}^{a}(x) & \equiv d^{a b c}\left(\bar{\psi} \gamma_{\mu} \lambda^{b} \psi\right)\left(\bar{\psi} \gamma_{\nu} \lambda^{c} \psi\right)(x) \\
\bar{l}_{\mu \nu}^{a}(x) & \equiv\left(\bar{\psi} \gamma_{\mu} \Lambda^{A} \psi\right)\left(\bar{\psi} \lambda^{a} \gamma_{\nu} \Lambda^{A} \psi\right)(x) \\
M_{\mu \nu}^{a}(x) & \equiv i f^{a b c}\left(\bar{\psi} \gamma_{\mu} \Lambda^{A} \lambda^{b} \psi\right)\left(\bar{\psi} \gamma_{\nu} \Lambda^{A} \lambda^{c} \psi\right)(x) \\
\bar{n}_{\mu \nu}^{a}(x) & \equiv d^{a b c}\left(\bar{\psi} \gamma_{\mu} \Lambda^{A} \lambda^{b} \psi\right)\left(\bar{\psi} \gamma_{\nu} \Lambda^{A} \lambda^{c} \psi\right)(x)
\end{aligned}
$$

We define the charge conjugation operator as in (6). Straightforward calculation shows that among the operators above $k_{\mu \nu}^{a}, l_{\mu \nu}^{a}$ and $\bar{l}_{\mu \nu}^{a}$ transform with $-\beta^{a b}$ i.e. they have different C-parity from the current's and thus do not appear in the OPE. The operators $n_{\mu \nu}^{a}$ and $\bar{n}_{\mu \nu}^{a}$ are symmetric Lorentz-tensors so following the general analysis of sec.(2) only four operators remain:

$$
j_{\mu}^{a}, \partial_{\mu} j_{\nu}^{a}, N_{\mu \nu}^{a}, M_{\mu \nu}^{a}
$$

\subsection{Perturbative calculation of the OPE}

In the following we will calculate the OPE coefficients up to the first nontrivial order in the coupling. Of course, a finite order perturbative approximation is not too conclusive from the nonlocal charge's point of view but later we will show that even this lowest-order approximation yields enough information to determine the exact OPE. The calculation is standard, the ultraviolet divergences are regulated by continuing from 2 to $d$ dimensions:

$$
\mathcal{L}=\bar{\psi} i \not \partial \psi-\frac{g_{0}^{2}\left(\mu^{2}\right)^{1-\frac{d}{2}}}{2}\left(\bar{\psi} \lambda^{a} \gamma_{\mu} \psi\right)^{2}
$$

The Feynman rules involve only one vertex with four legs. The two incoming 
and two outgoing fermion lines are contracted by the operator:

$$
-i g^{2}\left(\gamma_{\mu} \otimes \gamma^{\mu}\right)\left(\lambda^{a} \otimes \lambda^{a}\right)
$$

and in the many-flavor case the unit operator contracts the flavor indices. The Fourier-transform is defined as:

$$
\psi(p) \equiv \int \mathrm{d}^{d} x e^{i p x} \psi(x)
$$

The $\beta$-function of the theory is well known, and can be found in the literature, e.g. 22]:

$$
\beta(g) \equiv \mu \frac{d g}{d \mu}=\left(\frac{d}{2}-1\right) g-\frac{q}{2 \pi} g^{3}+\frac{q^{2}}{2 \pi^{2} n} g^{5}+\ldots
$$

We computed the 4-point function of the operator product $f^{a b c} j_{\mu}^{b}(x) j_{\nu}^{c}(0)$ as $x \rightarrow 0$ up to order $g^{2}$. The terms which are finite as $x \rightarrow 0$ yield the fourpoint function of the renormalized operator, $N_{\mu \nu}^{a}(0)$, the infinite terms can be identified with divergent coefficients times the four-point functions of the other operators: $j_{\mu}^{a}(0), \partial_{\mu} j_{\nu}^{a}(0), N_{\mu \nu}^{a}(0)$ and $M_{\mu \nu}^{a}(0)$. The summary of the calculation is the following. The amputated two-point function of $j_{\mu}^{a}(x)$ is:

$$
\left\langle\psi\left(p_{1}\right) j_{\mu}^{a}(x) \bar{\psi}\left(p_{2}\right)\right\rangle=\gamma_{\mu} \lambda^{a} e^{i\left(p_{1}-p_{2}\right) x}\left(1-g^{2} \frac{q N}{n \pi}+\mathcal{O}\left(g^{4}\right)\right) .
$$

We need the two-point function of the OPE at tree level:

$$
\begin{gathered}
\left\langle\psi\left(p_{1}\right) f^{a b c} j_{\mu}^{b}(x) j_{\nu}^{c}(0) \bar{\psi}\left(p_{2}\right)\right\rangle= \\
=\frac{q}{i \pi} \gamma_{\rho} \lambda^{a} \frac{g_{\mu \nu} x^{\rho}-x_{\mu} \delta_{\nu}^{\rho}-x_{\nu} \delta_{\mu}^{\rho}}{x^{2}}\left(1+\frac{i}{2}\left(p_{1}-p_{2}\right) x\right)+\mathcal{O}(|x|) .
\end{gathered}
$$

In view of (53) this can be written as

$$
\begin{gathered}
\left\langle\psi\left(p_{1}\right) f^{a b c} j_{\mu}^{b}(x) j_{\nu}^{c}(0) \bar{\psi}\left(p_{2}\right)\right\rangle= \\
=\frac{q}{i \pi} \frac{g_{\mu \nu} x^{\rho}-\left(\delta_{\mu}^{\rho} x_{\nu}+\delta_{\nu}^{\rho} x_{\mu}\right)}{x^{2}}\left\langle\psi\left(p_{1}\right)\left(j_{\rho}^{a}(0)+\frac{x^{\sigma}}{2} \partial_{\sigma} j_{\rho}^{a}(0)\right) \bar{\psi}\left(p_{2}\right)\right\rangle+\mathcal{O}(|x|) .
\end{gathered}
$$


Figure 1:
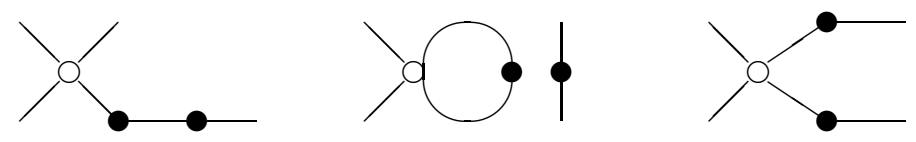

We now turn to the four-point function of the operator product. The tree-level matrix element is finite as $x \rightarrow 0$, it gives the corresponding correlation function of $-i N_{\mu \nu}^{a}(0)$. At the next order there are three different types of diagrams, these are shown in fig.17. The filled circles stand for the operator of the current and the empty circles denote the interaction vertex. As $x \rightarrow 0$ the graphs of the first type include the two-point function of the current-current OPE as a subgraph so we can substitute that result. It can be shown that the second graph is UV finite, and thus it yields the corresponding matrix element of $-i N_{\mu \nu}^{a}(0)$. The graphs belonging to the third type are UV divergent and give rise to logarithmic $x$-dependence. Since we expect that the operator $-i N_{\mu \nu}^{a}(0)$ will appear with leading coefficient 1 we calculated the difference of the matrix element of the operator product and $N_{\mu \nu}^{a}(0)$ (when dealing with diagrams of the third type).

A straightforward application of the Feynman rules shows that the following one-loop integral need to be calculated:

$$
f^{\rho \kappa}(p, q, x) \equiv \int \frac{\mathrm{d}^{d} k}{(2 \pi)^{d}} \frac{i k^{\rho}}{k^{2}} \frac{i(p+k)^{\kappa}}{(p+k)^{2}}\left(e^{i(k+q) x}-1\right) .
$$

It is easy to see that $p$ and $q$ do not affect the short-distance behavior of the integral, the derivatives with respect to these momenta are UV-convergent and vanishing as $x \rightarrow 0$. Therefore it is sufficient to consider the integral at arguments $p=q=0$. We obtain:

$$
f^{\rho \kappa}(0,0, x)=\frac{i}{8 \pi}\left(\frac{2 g^{\rho \kappa}}{d-2}-g^{\rho \kappa} \log \left(-\pi x^{2}\right)-\gamma_{E} g^{\rho \kappa}-2 \frac{x^{\rho} x^{\kappa}}{x^{2}}\right) .
$$

Here $-\gamma_{E}$ is the derivative of the $\Gamma$-function at 1 . The UV-divergent term is $x$-independent and is exactly equal to the divergent part of the unrenormalized 
Green's function of $N_{\mu \nu}^{a}(0)$. The $x$-independent UV divergences thus cancel when we replace $N_{\mu \nu}^{a}(0)$ with the renormalized operator. When putting together all the results we note that all the possible operators have nonzero four-point function at this order, so we can write our result as an operator equation :

$$
\begin{aligned}
f^{a b c} j_{\mu}^{b}(x) j_{\nu}^{c}(0)= & \frac{q}{i \pi} \frac{g_{\mu \nu} x^{\rho}-\delta_{\mu}^{\rho} x_{\nu}-\delta_{\nu}^{\rho} x_{\mu}}{x^{2}}\left(j_{\rho}^{a}(0)+\frac{x^{\sigma}}{2} \partial_{\sigma} j_{\rho}^{a}(0)\right)+(57) \\
& +\frac{1}{i}\left(1+\frac{g^{2} q}{2 \pi}\left(\frac{1}{2}+\gamma_{E}+\log \left(-\pi \mu^{2} x^{2}\right)\right)\right) N_{\mu \nu}^{a}(0)+(58) \\
& +\mathcal{O}\left(|x|^{1-0}\right) .
\end{aligned}
$$

There are two important facts to notice in this result. The only operator $M_{\mu \nu}^{a}(0)$ which could have also been present (in the many-flavor CGN) did not turn up at this order, now the question is whether it can be present with higher-order coefficient. In the next section we will show that this can not be the case: from the fact that its coefficient is zero at $g^{2}$-order follows that it vanishes. The other issue is the presence of the operator $N_{\mu \nu}^{a}(0)$. Notice that its coefficient is $x$-dependent, i.e. it is not constant. However the general analysis of the OPE showed that any antisymmetric tensoroperator which is independent of the current must have constant coefficient. From this it follows, that $N_{\mu \nu}^{a}(0)$ is not independent of the current and its derivative, we can erase it from the list of the possible operators. The precise statement is that the classical curl-free equation survives the quantization in the sense that the two operators remain linearly dependent. We give a functional integral argument for this in the appendix.

\subsection{Renormalization group for OPE coefficients}

We now turn to the question of the remaining operator, $M_{\mu \nu}^{a}(0)$. The OPE looks like:

$f^{a b c} j_{\mu}^{b}(x) j_{\nu}^{c}(0)=C_{\mu \nu}^{\rho}(x) j_{\rho}^{a}(0)+D_{S, \mu \nu}^{\sigma \rho}(x) S_{\sigma \rho}^{a}(0)+D_{A, \mu \nu}^{\sigma \rho}(x) A_{\sigma \rho}^{a}(0)+E_{\mu \nu}^{\sigma \rho} M_{\sigma \rho}^{a}(0),(59)$

where $S$ and $A$ mean the symmetrized and antisymmetrized derivative of the current (w.r.t. its Lorentz indices). We found in the general section that the 
coefficient $E$ must be constant. Since the currents are not renormalized, the most general renormalization which involves the four operators is:

$$
\left(\begin{array}{c}
j_{\mu}^{a} \\
S_{\mu \nu}^{a} \\
A_{\mu \nu}^{a} \\
M_{\mu \nu}^{a}
\end{array}\right)_{\text {Ren. }}=\left(\begin{array}{cccc}
1 & 0 & 0 & 0 \\
0 & 1 & 0 & 0 \\
0 & 0 & 1 & 0 \\
0 & 0 & Z_{M A} & Z_{M M}
\end{array}\right)\left(\begin{array}{c}
j_{\mu}^{a} \\
S_{\mu \nu}^{a} \\
A_{\mu \nu}^{a} \\
M_{\mu \nu}^{a}
\end{array}\right)_{0}
$$

When calculating the OPE coefficients perturbatively one obtains an explicit dependence of the Green's functions on the renormalization scale, $\mu$. The renormalization group equations ensure that the physical quantities do not depend on this scale if one also considers the implicit $\mu$-dependence of the couplings. By forming Green's functions from both sides of the OPE one can write RG equations for the OPE coefficients. Straightforward application of the operator $\mathcal{D} \equiv \mu \frac{d}{d \mu}$ to both sides yields:

$$
\begin{aligned}
0 & =\mathcal{D} C_{\mu \nu}^{\rho}(x) \\
0 & =\mathcal{D} D_{S, \mu \nu}^{\sigma \rho}(x) \\
0 & =\mathcal{D} D_{A, \mu \nu}^{\sigma \rho}(x)+E_{\mu \nu}^{\sigma \rho} \mathcal{D} Z_{M A}-E_{\mu \nu}^{\sigma \rho} \frac{Z_{M A}}{Z_{M M}} \mathcal{D} Z_{M M} \\
0 & =\mathcal{D} E_{\mu \nu}^{\sigma \rho}+E_{\mu \nu}^{\sigma \rho} \mathcal{D} \log \left(Z_{M M}\right) .
\end{aligned}
$$

The tensor structure of $E_{\mu \nu}^{\sigma \rho}$ is $\left(\delta_{\mu}^{\sigma} \delta_{\nu}^{\rho}-\delta_{\nu}^{\sigma} \delta_{\mu}^{\rho}\right) E$, from which the last equation yields:

$$
\mathcal{D} \log \left(E Z_{M M}\right)=0 .
$$

Suppose that we know the full perturbative series of $E$, and the anomalous dimension of $M_{\mu \nu}^{a}$ is also given as a power series in the coupling:

$$
\begin{aligned}
\mathcal{D} \log \left(Z_{M M}\right) \equiv \gamma_{M M} & =\eta_{1} g^{2}+\eta_{2} g^{4}+\ldots \\
E & =E_{0} g^{2 \alpha}\left(1+E_{1} g^{2}+E_{2} G^{4}+\ldots\right) .
\end{aligned}
$$

$E_{i}$ are independent of the space-time difference therefore they can not depend on $\mu$, either, since there is no other dimensionful quantity and the RG-derivative 
becomes very simple:

$$
\mathcal{D} \log \left(E Z_{M M}\right)=2 g \beta(g)\left(\frac{\alpha}{g^{2}}+E_{1}+\ldots\right)+\gamma_{M M} .
$$

The power expansion of the RG equation (65) then gives:

$$
\begin{aligned}
0 & =2 \beta_{0} \alpha+\eta_{1}+\mathcal{O}\left(g^{2}\right) \\
\alpha & =-\frac{\eta_{1}}{2 \beta_{0}} .
\end{aligned}
$$

The important consequence of this result is that the exponent of the leading perturbative behavior of the coefficient $E$ is known if we calculate the 1-loop $\beta$ - and $\gamma$-functions. Having calculated this number, $\alpha$ we obtain the following information. If $\alpha$ is not a nonnegative integer then the only possible solution for the RGE is $E=0$ since one can not obtain this kind of behavior in perturbation theory. If $\alpha$ is a nonnegative integer then it shows the order of perturbation theory where one first obtains a nonzero result when calculating the OPE coefficient. This is most informative in the case when one calculates the coefficient up to $\alpha$ th order and obtains zero; then conclusion is then that it remains zero to all orders.

It is a straightforward exercise to calculate $\gamma_{M M}$ at first order, we get: $\eta_{1}=\frac{q}{\pi}$. From this we obtain $\alpha=1$. In the previous section we found that $E=0$ at the order of $g^{2}$ which shows that the OPE coefficient remains zero to any order. Thus the operator $M_{\mu \nu}^{a}(0)$ can not enter the OPE.

\subsection{Summary}

The perturbative calculation in the one-flavor and many-flavor CGN model showed that in our OPE only the color current and its derivative is present. With the help of (58) and (76) we can determine the scalar OPE coefficients:

$$
\begin{aligned}
\gamma_{2} & =-\gamma_{1}=\lambda=-\frac{q}{i \pi} \\
\delta_{A} & =i\left(\frac{2}{g^{2}}-\frac{q}{\pi}\left(\log \left(-\pi \mu^{2} x^{2}\right)+\gamma_{E}+\frac{1}{2}\right)\right) .
\end{aligned}
$$


This can be transformed into a more transparent form if we use the asymptotic freedom and introduce the ( $x$-dependent) running coupling:

$$
\frac{1}{\bar{g}^{2}}=-\frac{q}{2 \pi} \log \left(-\Lambda^{2} x^{2}\right)-\frac{q}{\pi n} \log \left(-\log \left(-\Lambda^{2} x^{2}\right)\right)+\frac{q}{2 \pi} \log \left(-\mu^{2} x_{0}^{2}\right)+\text { const. }
$$

Here $\Lambda$ is the dimensional transmutation parameter. Then the OPE coefficient (in $\overline{M S}$ scheme) is:

$$
\delta_{A}=\frac{q}{i \pi} \log \left(-\Lambda^{2} x^{2}\right)+\frac{2 q}{i \pi n} \log \left(-\log \left(-\Lambda^{2} x^{2}\right)\right)+\frac{q}{\pi i}\left(\frac{1}{2}-\log (4)\right) .
$$

In the one-flavor CGN-model the existence of the non-local charge is essentially ensured by the kinematics (similarly to the $O(n) \sigma$-models): simply there is no extra operator which could appear in the OPE. This is a well-known result [7]. The novelty in our treatment of the MCGN is that in this case the general OPE does not yield enough information and we had to use a dynamical argument to rule out the extra operator which could have been present a priori.

\section{Conclusion}

In the present paper we discussed two subjects. By reviewing previous results obtained in integrable models we determined a general sufficient criterion for the existence of conserved non-local charges and Yangian algebra in a class of $2 \mathrm{~d}$ quantum field theories. We showed the crucial role of the current-current OPE and pointed out how the most general form (allowed by general principles) of the OPE-coefficients ensures the existence and conservation of the non-local charge provided there are no "extra" operators in the Wilson expansion. It turned out that the properties and the coefficients of these operators are also highly constrained which may help in dealing with them in concrete theories.

We employed these general results to compare the one- and multiflavor CGN model. As it was found previously $[7$ the one-flavor model belongs to the trivial case of the analysis: there are too few operators and the algebra of 
the currents must close on themselves. This is not the case in the multiflavor model where the flavor-space increases the number of degrees of freedom and the new operators could in principle invalidate the conservation of the nonlocal charge. We had to take a closer look at the OPE for which we used renormalized perturbation theory improved by renormalization group. The vanishing of the extra operator at one loop level was promoted by the RG-arguments to all orders thus answering the crucial question: the OPE of the current closes on themselves in the multiflavor model too.

Our results show that although the particle spectrum of the one- and the multiflavor chiral model is different 19 nevertheless the same Yangian symmetry algebra is realized. This fact points to the advantage of the operator-algebra approach: one can study the symmetry structure without having to refer to the spectrum of the theory.

The proof of the existence of the Hopf-algebra symmetry generated by non-local charges is a first step in proving the integrability of a model. We think that the consequences on the mass spectrum, S-matrix and correlation functions can be analyzed similarly to the models studied previously, e.g [4, 6, [1.

I wish to thank J. Balog for the introduction into this field and his continuous support and gratefully acknowledge discussions with P. Forgács.

\section{Appendix: The curl-free equation}

As it is discussed in [19, 20] the current-current interaction of the multiflavor CGN model can be replaced by an equivalent vector-boson mediated one:

$$
S=\int d^{2} x\left(\bar{\psi} i \not \partial \psi-A_{\mu}^{a} j^{\mu a}+\frac{1}{2 g^{2}} A_{\mu}^{a} A^{\mu a}\right)
$$

The functional integral (which should be defined in Euclidean space by Wick rotation) over the auxiliary field $A_{\mu}^{a}$ is purely Gaussian thus the quantum equivalence clearly holds. On the other hand one can perform the fermionic integral 
first; leading to the effective action of the gauge fields (which may be viewed as a classical background). The breakdown of the axial invariance of the effective action yields the axial anomaly equation:

$$
D_{\mu} j_{5}^{\mu}=\frac{N q}{2 \pi n} \epsilon^{\mu \nu} F_{\mu \nu}
$$

Here $j_{5}^{\mu a}=-\epsilon^{\mu \nu} j_{\nu}^{a}$ is the axial current in $2 \mathrm{~d}$ and $F_{\mu \nu}$ is the field-strength tensor corresponding to $A_{\mu}^{a}$. This equation is obtained in any gauge-invariant regularization. By considering this equation inside a correlation function involving fermionic fields one can perform the integral over the vector field first and an operator equation in the original purely fermionic model is obtained. Note that the integral does not cause any problem in the quadratic part of $F_{\mu \nu}$ because of the antisymmetry of the color structure constant, $f^{a b c}$. Evaluating the functional integral over the vector field then yields:

$$
\left(1+g^{2} \frac{q N}{\pi n}\right)\left(\partial_{\mu} j_{\nu}^{a}-\partial_{\nu} j_{\mu}^{a}\right)+2 g^{2}\left(1+g^{2} \frac{q N}{2 \pi n}\right) i f^{a b c} j_{\mu}^{b} j_{\nu}^{c}=0 .
$$

Note that our vector-boson theory does not require a gauge-invariant scheme and by a suitable finite renormalization we can change the anomaly equation such that the classical curl-free equation is not modified. One can therefore define the quantum CGN-model keeping

$$
\partial_{\mu} j_{\nu}^{a}-\partial_{\nu} j_{\mu}^{a}+2 g^{2} i f^{a b c} j_{\mu}^{b} j_{\nu}^{c}=0 .
$$

Whether $(76)$ or $(77)$ is chosen as the renormalization scheme the lesson is the same: the operator $N_{\mu \nu}^{a}$ is not independent of the antisymmetrized derivative of the current.

\section{References}

[1] D. Bernard and A. Leclair, Nucl. Phys B399 (1993) 709.

[2] H.J. de Vega, H. Eichenherr and J.M. Maillet Comm.Math.Phys. 92 (1984) 507. 
[3] H.J. de Vega, H. Eichenherr and J.M. Maillet Nucl. Phys. B240 (1984) 377.

[4] M. Lüscher, Nucl. Phys. B135 (1978) 1.

[5] D. Bernard, Comm.Math.Phys. 137 (1991) 191.

[6] E.Abdalla and A. Lima-Santos, Rev.Bras.Fis 12 (1982) 293.

[7] E. Abdalla, M.C.B. Abdalla and K.D. Rothe Nonperturbative methods in two-dimensional quantum field theory Singapore, World Scientific (1991)

[8] D. Buchholz and J.T. Lopuszanski Lett. Math. Phys 3 (1979) 175.

[9] D. Buchholz J.T. Lopuszanski and Sz. Rabsztyn Nucl. Phys. B263 (1986) 155.

[10] A. LeClair and F.A. Smirnov Int. J. Mod. Phys. A7 (1992) 2997.

[11] D. Bernard, Talk given at the "Integrable Quantum Field Theories" conference held at Come, Italy, September 13-19, 1992

[12] A. Belavin Phys. Lett. B283 (1992) 67.

[13] M. Lüscher Unpublished notes

[14] R.F. Streater and A.S. Wightman, PCT, Spin and Statistics, and All That Benjamin, New York, 1964

[15] D.J. Gross and A. Neveu, Phys. Rev. D10 (1974) 3235.

[16] T.L. Curtright and C.K. Zachos Phys. Rev. D24 (1981) 2661.

[17] P.K. Mitter and P.H. Weisz, Phys. Rev. D8 (1973) 4410.

[18] E. Witten Nucl. Phys. B145 (1978) 110.

[19] A. Polyakov and P.B. Wiegmann, Phys. Lett 131B (1983) 121.

[20] C. Destri and H.J. de Vega, Phys. Lett. 201B (1988) 245.

[21] C.Destri, Phys. Lett. 210B (1988) 173.

[22] P.Forgács, S.Naik and F. Niedermayer, Phys. Lett. 283B (1992) 282. 